\title{
INFECTIOUS DISEASES, NSW: MARCH 1999
}

\section{TRENDS}

\section{Legionnaires disease}

A cluster of patients with pneumonia due to Legionnaires disease was reported in February this year (Figure 3 and Table 1). The causative organism, Legionella pneumophila, commonly develops in collections of warm water and may infect people who inhale aerosols produced by the cooling towers air conditioning systems in large buildings. The elderly, people who are immunosuppressed and smokers are at increased risk for the disease. Between 33 and 105 cases are reported yearly in NSW.

Seven cases of pneumonia due to Legionella pneumophila serogroup one were reported in people living in different parts of western Sydney, with onset dates between the 7th and 20th of February. Four of these cases were confirmed by positive tests for urinary antigen, and three were confirmed by sputum culture. All seven patients required hospitalisation, four in intensive care. All have recovered.

Western Sector Public Health Unit investigators gathered exposure histories for each case, either by direct interview or, for two cases who were too ill for questioning, by interviewing close relatives. No single common exposure was found for the cases.

Three cases reported visiting the vicinity of a shopping complex, and two cases reported visiting a club. However, given the popularity of these institutions in the locality, no definite causal link could be drawn. As a precaution, six cooling towers within a one kilometre radius of the shopping complex and the two cooling towers at the club were inspected and sampled and it was advised that they be cleaned. While none of the samples from around the shopping complex were positive for Legionella pneumophila, one of the cooling towers at the club returned a high positive count that was probably associated with the exhaustion of biocide.

Subsequent tests at the Institute of Clinical Pathology and Medical Research (ICPMR) laboratory, Westmead, on the sputum isolates from the three culture-confirmed patients found that they had different DNA fingerprinting patterns, suggesting that the cases were unrelated.

Such reports of apparently sporadic cases of Legionnaires disease serve as a reminder of the importance of minimising bacterial growth by careful inspection, cleaning and maintenance of cooling towers by building owners. For more information contact your local council or public health unit.

\section{Arbovirus surveillance}

During January and February, the NSW Arbovirus Surveillance and Vector Monitoring Program detected Kunjin virus in mosquitoes collected in the Griffith and Leeton areas of Greater Murray.

Kunjin is a flavivirus whose primary hosts are believed to be water birds who transmit it to mosquitoes. It is closely related to the Murray Valley encephalitis virus, although it causes a milder illness. Kunjin has been shown to cause a febrile polyarthritic illness and occasionally mild encephalitis.

Following the detection of the virus, disease prevention messages were intensified in the Leeton and Griffith areas and general practitioners were informed about the need to consider Kunjin virus when investigating suspected arboviral disease.

Flocks of chickens in selected sites across rural NSW are tested routinely in summer months to see if they have seroconverted, which would mean they had been infected with flaviviruses. No Kunjin virus activity was detected in the sentinel chickens located near Griffith and Leeton, and no acute cases of human disease due to Kunjin have been detected. This suggests that, although the virus was active in the area, the level of virus had not increased sufficiently to cause human disease.

The arbovirus season in the Greater Murray was characterised by high numbers of mosquitoes and a large number of cases of Ross River virus disease, particularly during November and December 1998. Residents are strongly advised to take appropriate personal protective measures (cover up, wear insect repellents, avoid mosquito exposures particularly at dawn and dusk) to reduce risk of infection.

For more information about the Arbovirus Surveillance and Vector Monitoring Program, visit the web site at www.arbovirus.health.nsw.gov.au. it: 


\section{FIGURE 3}

REPORTS OF SELECTED INFECTIOUS DISEASES, NSW, JANUARY 1994 TO FEBRUARY 1999, BY MONTH OF ONSET

These are preliminary data: case counts in recent months may increase because of reporting delays

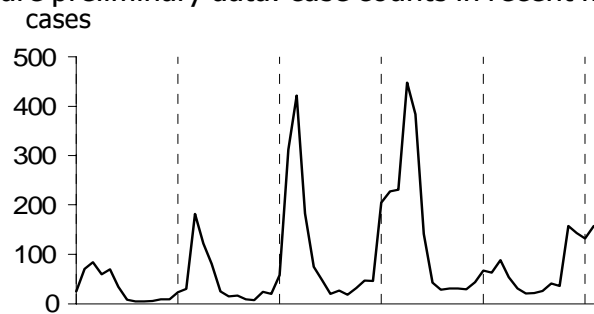

Arbovirus
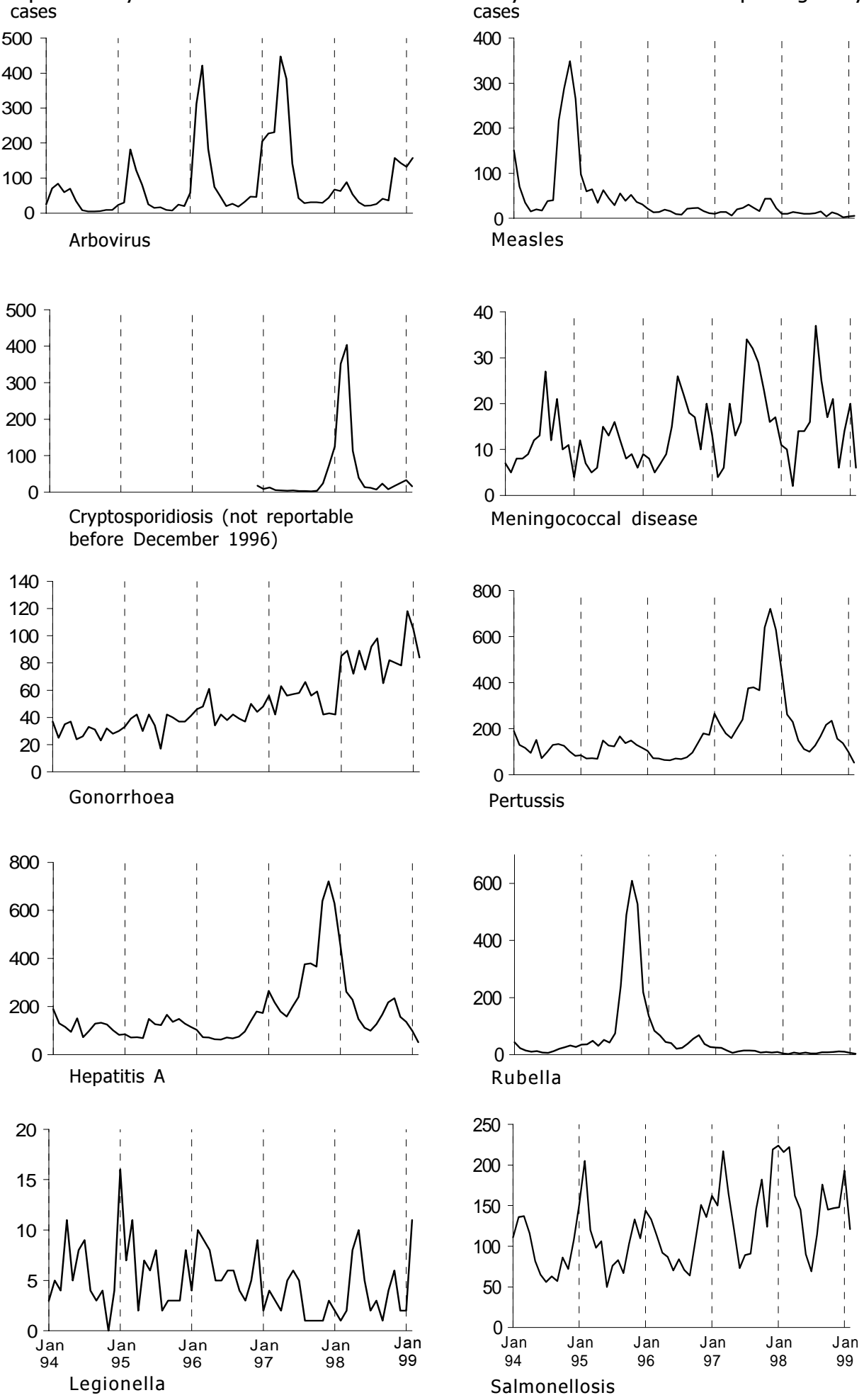


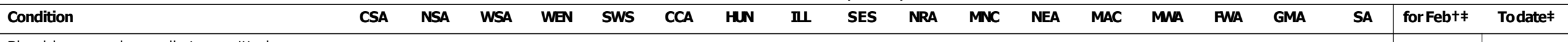

Blood-borne and sexually transmitted AIDS

HIV infection*

Hepatitis B: acute viral*

Hepatitis B: other*

Hepatitis C: acute viral

Hepatitis C: other*

Hepatitis D: unspecified*

Hepatitis, acute viral (not otherwise specified) Chlamydia (genital)

Gonorrho

$\begin{array}{rr}2 & 1 \\ - & - \\ - & - \\ 56 & 34 \\ - & - \\ 58 & 42 \\ - & - \\ - & - \\ 11 & 7 \\ 22 & 9 \\ 6 & 5\end{array}$

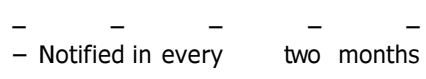

Vector-borne

Arboviral infection*

$4 \quad 5$

- Notified in every two months

Malaria*

\begin{tabular}{ll}
4 & 5 \\
2 & 5 \\
\hline
\end{tabular}

$\begin{array}{rr}- & \\ - & \\ - & \\ 6 & 47 \\ - & \\ 18 & 80 \\ - & \\ - & \\ 13 & 37 \\ 2 & 41 \\ - & 11\end{array}$

$\begin{array}{rr}1 & 2 \\ - & - \\ 2 & 1 \\ 47 & 2 \\ - & - \\ 80 & 36 \\ - & - \\ - & - \\ 37 & 12 \\ 41 & 3 \\ 11 & 3\end{array}$

\begin{tabular}{rrr}
2 & - & - \\
\hline & - & - \\
2 & - & - \\
- & - & 4 \\
36 & 28 & 1 \\
- & - & - \\
- & - & - \\
12 & 3 & 5 \\
3 & 2 & 1 \\
3 & 1 & 2
\end{tabular}

Zoonoses

Brucellosis*
Leptospirosis*

Q fever*

\section{Respiratory and othe}

Blood lead level

Legionnaires' disease

Leprosy

Meningococcal infection (invasive)

Mycobacterial tuberculosis

Vaccine-preventable

Adverse event after immunisation

$H$. influenzae $b$ infection (invasive)

Measles

Mumps*

Pertussis

Rubella*

Faecal-oral

Botulism

Cholera*
Cryptosporidiosis

Cryptospo

Food-borne illness (not otherwise specified)

Gastroenteritis (in and institution)

Haemolytic uraemic syndrome

Hepatitis A

Hepatitis E

Salmonellosis (not otherwise specified)*

Typhoid and paratyphoid

Verotoxin producing $E$. coll

* lab-confirmed cases only

$\begin{array}{llll}5 & 2 & 1 & - \\ - & - & -\end{array}$

\begin{tabular}{rrr}
21 & 7 \\
$-\quad 1$ & 2 \\
\hline
\end{tabular}

2

$\begin{aligned} \text { CSA } & =\text { Central Sydney Area } \\ \text { NSA } & =\text { Northern Sydney Are }\end{aligned}$

WSA $=$ Western Sydney Area

\section{WEN $=$ Wentworth Area}

SWS $=$ South Western Sydney Area

CCA $=$ Central Coast Area

HUN $=$ Hunter Area
ILL = Illawarra Area

SES = South Eastern Sydney Area

NRA $=$ Northern Rivers Area

$\mathrm{MNC}=$ North Coast Area
$\mathrm{NEA}=$ New England Area

$\begin{array}{rllllllll}- & - & - & - & - & - & - & - & 1 \\ 4 & 2 & - & 3 & 4 & - & 7 & 1 & 1 \\ - & - & 6 & 1 & - & - & 1 & - & 2 \\ - & - & - & - & - & - & - & - & 2 \\ - & - & 2 & - & 1 & - & 1 & - & 5 \\ 4 & 5 & 6 & - & 2 & - & - & - & 6\end{array}$

$\begin{array}{rr}- & - \\ - & - \\ 1 & - \\ 2 & 3 \\ - & - \\ 5 & 25 \\ - & - \\ - & - \\ - & 6 \\ - & 2 \\ 1 & 2\end{array}$

-
-
-
7
4
3
-
-
3
5
5

-
-
-
1
-
29
-
-
-
-

9
-
4
190
5
486
-
-
129
94
39

55
16
9
335
11
995
-
-
275
208
70

2

2
8

23

138 $\begin{array}{r}2339 \\ 60 \\ \hline\end{array}$ 